\title{
LAWNGTLANG ZOPHEI SWADESH LIST
}

\author{
July, 2019 \\ Samson Lotven, Zai Sung, James C. Wamsley, Kelly Berkson ${ }^{l}$ \\ ${ }^{1}$ Indiana University Department of Linguistics
}

\section{INTRODUCTION}

This paper presents a preliminary 100-item Swadesh word list for Lawngtlang Zophei. Zophei or Zyphe (ISO 639-3 ZYP) belongs to the Maraic branch of Kuki-Chin within the Tibeto-Burman language family (Eberhard et. al, 2019). Lawngtlang is a Zophei village in the Southeastern corner of the Zophei-speaking area in Thantlang Township, Chin State, Myanmar. Lawngtlang Zophei is considered to be part of the Lower (western) dialects of Zophei. The word list comes from the intuitions of our co-author Zai Sung, a 22-year-old native speaker born in Lawngtlang and currently living in Indianapolis, Indiana. She also speaks Hakha Chin and English, as well as having some familiarity with Burmese (the language of education in Myanmar). The Lawngtlang Zophei consonant and vowel inventories are as follows (for consistency, voiceless affricates and sonorants are indicated with a superscripted $\langle\mathrm{h}\rangle$ despite phonetic differences) ${ }^{1}$ :

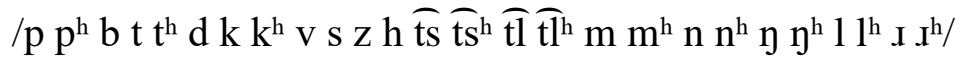

/ii i yy ee e $\varnothing \varnothing$ aa a uu u it mu ie yo ei au/

It is important to note that Zophei is a tone language. Our analysis of Zophei tone is in its infancy, however, and so in the present list we focus on segmental content and leave tone to be addressed in future work. Section 2 includes a 99-item Lawngtlang Zophei word list based on Swadesh's (1971) 100-word list ('fingernail' and 'claw' have identical translations and were collapsed into a single entry).

\section{WORD LIST}

\begin{tabular}{|c|l|l|}
\hline & Gloss & Lawngtlang Zophei \\
\hline 1. & I & ka maa \\
\hline 2. & you & na maa \\
\hline 3. & we & kaa nii \\
\hline 4. & this & he \\
\hline 5. & that & $\mathrm{k}^{\mathrm{h}}$, $\widehat{\mathrm{saa}}^{2}$ \\
\hline 6. & who? & a huu \\
\hline 7. & what? & zee \\
\hline 8. & not & bee \\
\hline
\end{tabular}

\footnotetext{
${ }^{1}$ An /ui/ diphthong is also attested in a single token ([bui ba] 'to be disordered'). Hakha Chin buai-bai 'very busy, confused, be disordered' (VanBik, personal communication, July 13, 2019).

${ }^{2} k h e h$ refers to objects in view of the speaker, $t$ sah is used for objects out of sight.
} 
Indiana Working Papers in South Asian Languages and Cultures

\begin{tabular}{|c|c|c|}
\hline 9. & all & don tii \\
\hline 10. & many & $\mathrm{l}^{\mathrm{h}} \mathrm{uH}$ \\
\hline 11. & one & say khe \\
\hline 12. & two & say $y^{\text {hin }}$ \\
\hline 13. & big & len \\
\hline 14. & long & $s \varnothing \emptyset$ \\
\hline 15. & small & $\mathrm{ts}^{\mathrm{t}} \mathrm{ie}$ \\
\hline 16. & unmarried woman & ley sau \\
\hline 17. & unmarried man, boyfriend & sa tTee \\
\hline 18. & person & tsang sau \\
\hline 19. & fish & yau \\
\hline 20. & bird & pa vau \\
\hline 21. & $\operatorname{dog}$ & yy \\
\hline 22. & louse & $\mathrm{I}^{\mathrm{h}} \mathrm{i}$ \\
\hline 23. & tree, wood & $\mathrm{t}^{\mathrm{h}} \mathrm{in}$ \\
\hline 24. & seed & t $\overrightarrow{S i i}$ \\
\hline 25. & leaf & t $\widetilde{s i t} n^{\mathrm{h}} \mathrm{au}$ \\
\hline 26. & root & $\mathrm{I}^{\mathrm{h}} \mathrm{an}$ \\
\hline 27. & bark & tsey \\
\hline 28. & skin & vin \\
\hline 29. & flesh & yen \\
\hline 30. & blood & $\mathrm{t}^{\mathrm{h}} \ddot{\mathrm{it}}$ \\
\hline 31. & bone & ItuH \\
\hline 32. & grease & $\mathrm{t}^{\mathrm{h}} \varnothing \emptyset$ \\
\hline 33. & egg & aa tyy \\
\hline 34. & horn, angle, corner, side & kii \\
\hline 35. & tail & la minee \\
\hline 36. & feather & ma thau \\
\hline 37. & hair (on head) & say \\
\hline 38. & head & lut \\
\hline 39. & ear & nau \\
\hline 40. & eye & $\mathrm{mi}$ \\
\hline 41. & nose & $\mathrm{n}^{\mathrm{h}} \mathrm{a} \mathrm{k} \mathrm{k}^{\mathrm{h}} \mathrm{uu}$ \\
\hline 42. & mouth & $\mathrm{kau}$ \\
\hline 43. & tooth & hau \\
\hline 44. & tongue & lyy \\
\hline 45. & fingernail, claw & ki pa tin \\
\hline 46. & foot (lower leg) & $\mathrm{p}^{\mathrm{h}} \mathrm{ee}$ \\
\hline 47. & knee & $\mathrm{k}^{\mathrm{h}} \mathrm{u}$ \\
\hline 48. & hand & $\mathrm{ki}$ \\
\hline 49. & belly & puu \\
\hline 50. & neck & Ion \\
\hline 51. & breasts & mei mei \\
\hline
\end{tabular}




\begin{tabular}{|c|c|c|}
\hline 52. & heart, liver & luy \\
\hline 53. & liver & $\mathrm{t}^{\mathrm{t}}$ in \\
\hline 54. & drink & $\operatorname{din}$ \\
\hline 55. & eat & ee \\
\hline 56. & bite & tøळ \\
\hline 57. & see & $\mathrm{m}^{\mathrm{h}} \mathrm{u}$ \\
\hline 58. & hear & $\mathrm{t}^{\mathrm{h}} \mathrm{yy}$ \\
\hline 59. & know, understand & bii \\
\hline 60. & sleep, lie down & $\mathrm{i}$ \\
\hline 61. & die & $\mathrm{t}^{\mathrm{t}} \mathrm{i} \dot{\mathrm{t}}$ \\
\hline 62. & kill & noy \\
\hline 63. & swim & tyy $1 \varnothing \varnothing$ \\
\hline 64. & fly & $\mathrm{z} \emptyset \emptyset$ \\
\hline 65. & go, walk & sii \\
\hline 66. & come & va sii \\
\hline 67. & sit & tøळ \\
\hline 68. & stand, rise & $\mathrm{t}^{\mathrm{h}} \mathrm{uu}$ \\
\hline 69. & give & pii/pi \\
\hline 70. & say & $\mathrm{ts}^{\mathrm{h}} \mathrm{in}$ \\
\hline 71. & sun, day & nïi \\
\hline 72. & moon & tThau pau \\
\hline 73. & star & aa sï \\
\hline 74. & water & tyy \\
\hline 75. & rain & $\mathrm{k}^{\mathrm{h}} \mathrm{ua}$ suu \\
\hline 76. & stone, rock & $\operatorname{lun}$ \\
\hline 77. & sand & sa dii \\
\hline 78. & earth, dirt & lyy \\
\hline 79. & cloud & mee din \\
\hline 80. & smoke, steam & $\mathrm{k}^{\mathrm{h}} \mathrm{ut}$ \\
\hline 81. & fire & mee \\
\hline 82. & ash & mee $\mathrm{k}^{\mathrm{h}} \mathrm{ut}$ \\
\hline 83. & burn & kay \\
\hline 84. & path, road, way & lan \\
\hline 85. & mountain & tTan \\
\hline 86. & red & sey \\
\hline 87. & green, alive, fresh & a $\mathrm{s}^{\mathrm{h}}$ in \\
\hline 88. & yellow & men \\
\hline 89. & white & Iay \\
\hline 90. & black & von \\
\hline 91. & night & zey \\
\hline 92. & hot & si \\
\hline 93. & cold & tŝa kau \\
\hline 94. & full, complete & tTin \\
\hline
\end{tabular}


Indiana Working Papers in South Asian Languages and Cultures

\begin{tabular}{|c|l|l|}
\hline 95. & new, fresh & $\mathrm{t}^{\mathrm{h}} \mathrm{aa}$ \\
\hline 96. & good & $\mathrm{tS}^{\mathrm{h}} \mathrm{a}$ \\
\hline 97. & round & puy \\
\hline 98. & dry & $\mathrm{\iota \emptyset}$ \\
\hline 99. & name & min \\
\hline
\end{tabular}

\section{REFERENCES}

Eberhard, D. M., Simons, G. F., and Fennig, C. D. (eds.) (2019) "Ethnologue: Languages of the World. Twenty-second edition" (SIL International, Dallas, Texas), Online version: http://www.ethnologue.com.

Swadesh, M. (1971) The origin and diversification of language (Aldine-Atherton, Chicago, Illinois), pp. 1-350.

VanBik, K. (2019, July 13). Email. 